品

SSCL-688

October 1994

Distribution Category: 400

A. Maschke

\title{
Final Report of the SSC Electrodesorption Studies
}

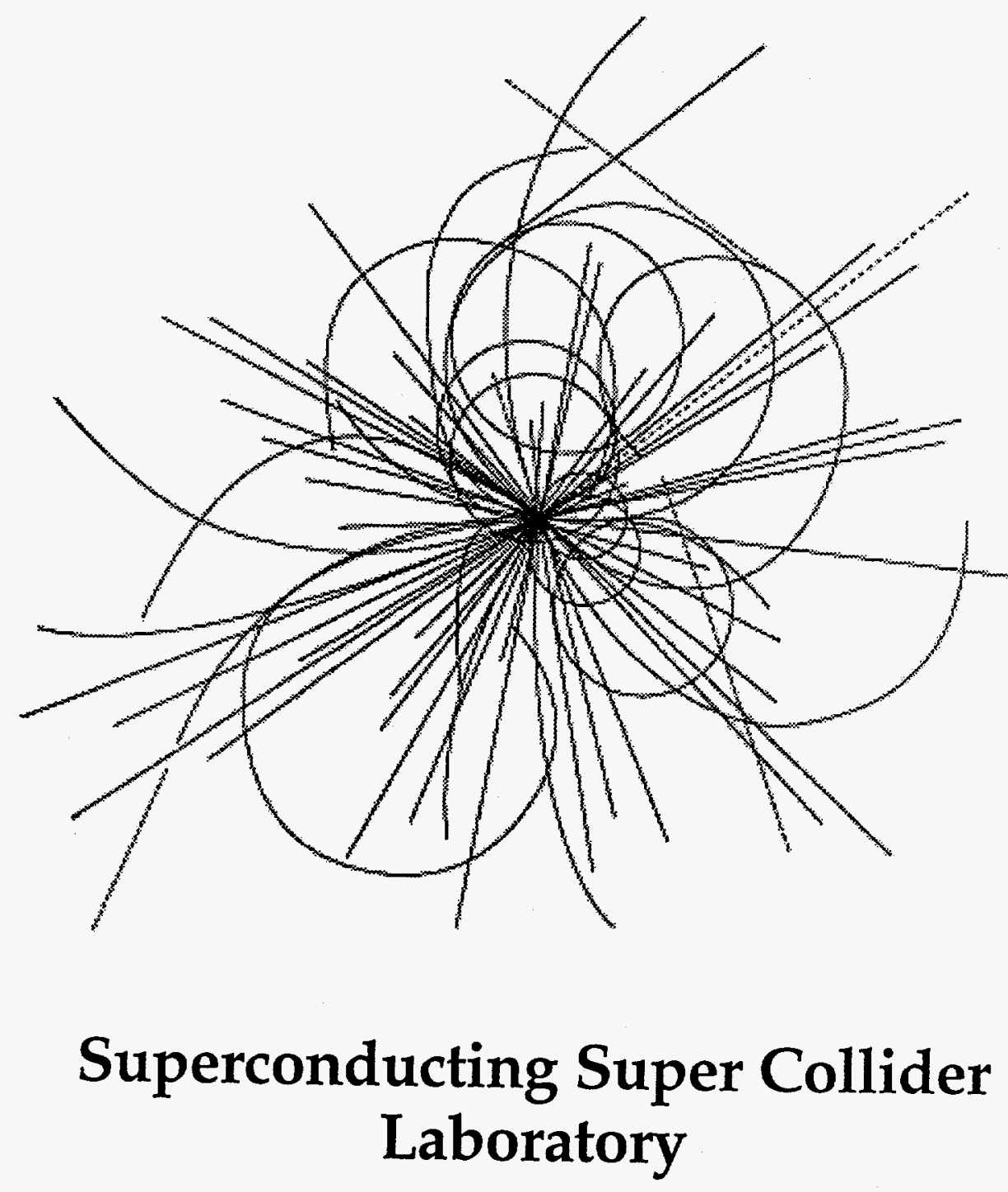




\section{Disclaimer Notice}

This report was prepared as an account of work sponsored by an agency of the United States Government. Neithar the United States Government or any agency thereof, nor any of their employees, makes any warranty, express or implied, or assumes any legal liability or responsibility for the accuracy, completeness, or usefulness of any information, apparatus, product, or process disclosed, or represents that its use would not infringe privately owned rights. Reference herein to any specific commercial product, process, or service by trade name, trademark, manufacturer, or otherwise, does not necessarily constitute or imply its endorsement, recommendation, or favoring by the United States Government or any agency thereof. The views and opinions of authors expressed herein do not necessarily state or reflect those of the United States Government or any agency thereof.

Superconducting Super Collider Laboratory is an equal opportunity employer. 


\section{DISCLAIMER}

Portions of this document may be illegible in electronic image products. Images are produced from the best available original document. 
SSCL-688

\title{
Final Report of the SSC Electrodesorption Studies
}

\author{
A. Maschke \\ Superconducting Super Collider Laboratory* \\ 2275 North Highway 77 \\ Waxahachie, TX 75165
}

October 1994

"Operated by the Universities Research Association, Inc., for the U.S. Department of Energy under Contract No. DE-AC35-89ER40486.

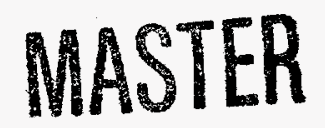




\subsection{INTRODUCTION}

Electrodesorption studies at the Superconducting Super Collider (SSC) were part of a larger program to try to understand what would be going on inside the SSC beam tube-for example, how much of the beam tube surface would be exposed to desorbing radiation. Since the incident synchrotron radiation lands on a few-millimeter-wide strip along the beam tube, it is possible that only a very small fraction of the surface would be exposed if the radiation were largely absorbed without rescatter. Therefore, we wanted information about scattering and absorption from various candidate surfaces.

The earlier electroplating studies that were carried out by the Central Design Group emphasized high values of the conductivity at $4 \mathrm{~K}$. We wanted to find out if there were other electroplating techniques that might give adequate conductivity but a smaller cross section for hydrogen desorption. This would include looking for other coatings that might reduce the desorption. Gold was often suggested as a possibility.

\subsection{EXPERIMENTAL SETUP}

The experimental setup for the first round of experiments was designed to expose room-temperature copper samples, although we did have the capability of cooling the samples to near liquid nitrogen $\left(\mathrm{LN}_{2}\right)$ temperature, albeit without proper measurement. The vacuum chamber consisted entirely of commercially available conflat seal pieces. The two main elements were a 4-way and a 6-way cross, coupled together. The 4-way cross had a VG quadrupole mass analyzer Model 100D on top and an $80 \mathrm{l} / \mathrm{s}$ bakeable turbo on the bottom. The turbo pump had a solid copper gasket with a small hole in its center that restricted the pumping speed to about $7.5 \mathrm{l} / \mathrm{s} \mathrm{N} \mathrm{N}_{2}$ equivalent. The horizontal member of the cross had an all-metal variable leak, usually used to admit gas from a calibrated leak. The fourth arm of the cross was attached to the 6-way cross, assuring that the gas entering from the chamber in which the electrodesorption occurred and the gas from the calibrated leak were symmetrically placed with respect to the vacuum pump.

The 6-way cross had a 0-1000 V Kimble Physics flood gun attached to the arm opposite the one connected to the 4-way cross. The bottom arm had a 500-A copper feedthrough attached to it. The removable samples were slid into place along an alignment groove machined into the copper feedthrough. This assured that each sample would be in the same place with respect to the electron gun and the rest of the apparatus. The samples were admitted to the vacuum system through an airlock attached to the horizontal arms. Opposite the airlock was a window that was used to facilitate placement of the sample onto the copper pedestal. The rubber O-ring on the airlock valve was the only non-metal gasket on the system. The top arm of the cross had a thin, stainless steel screen capable of being moved up and down to shield or expose the sample. The screen came down just a couple of millimeters in front of the sample. It also had a $0.25 \mathrm{~mm}$ wire stretched across the two support rods that supported the screen. The entire array was isolated from ground to allow current measurement of the screen and wire. The movable wire allowed us to measure the beam profile.

\subsection{EXPERIMENTAL PROCEDURES}

We begin by describing the procedures used in performing the electrodesorption measurements at room temperature.

An airlock for sample entry was important in allowing us to make repeatable measurements. Letting the electron gun and residual gas analyzer (RGA) up to atmosphere each time a new sample was introduced meant that they had to be carefully conditioned to assure that they would provide repeatable performances. An even more important consideration was the amount of dynamic background present while the beam was on. When the electron beam irradiated a sample, the pressure would rise immediately, then start to fall rapidly as the exposed surface was cleaned up. However, even with hours of exposure, the background would not return to the level before the beam was turned on. This dynamic background came from radiation emitted from the sample and falling on the surrounding stainless steel vacuum chamber. This background 
could be brought down by days of operation at high current. In order to make accurate measurements of the desorption from the sample, it was always necessary to subtract the dynamic background. Therefore, the accuracy of the desorption measurements was always improved by having the dynamic background as small as possible. It was also important that the dynamic background remain constant during the course of the experiment.

The movable screen in front of the sample was an important addition to the experimental procedures. This allowed us to make sure that the electron-gun current was the same as it was in previous runs. In addition, we could measure the dynamic background before the start of an exposure.

The samples were all biased at $-18 \mathrm{~V}$. It was important to make sure that the secondary electrons emitted from the sample did not come back and hit it. In order to obtain quantitative results from the electrodesorption experiments it is necessary to know the electron beam energy and density distribution of the sample. This is impossible if the sample is biased positively. Only a few volts of negative bias was necessary to assure good performance. The $-18 \mathrm{~V}$ choice was rather arbitrary.

The goal of the electrodesorption measurements was to determine the amount of gas desorbed per unit surface area for a given number of electrons per unit surface area. We did not want to have to rely on making an absolute calibration of the RGA, which is difficult and must be repeated often because the calibration can easily change. The method of calibration we used was to measure the response of the RGA to a sample exposure, then compare this with the response to a calibrated leak whose size was roughly the same as the amount of gas from the electrodesorption. We had two calibrated leaks for hydrogen, the larger of which brought the vacuum up to about $2-3 \times 10^{-7}$ Torr, which was well within the linear response region for the RGA. The smaller leak was about 100 times smaller, and allowed us to verify that the RGA response was linear over the region of interest.

An important element of the calibration procedure is the assumption that the RGA response to a given amount of desorbed gas will be the same as that from the calibrated leak. In our setup for room-temperature measurements, we guaranteed the correct readings by making sure that the two sources of gas were equidistant from the vacuum pump and the RGA.

In order to obtain accurate and repeatable results during the course of an exposure, it was important that the only variable in the system during that time was the gas desorption coming from direct electron impact on the sample. There were several problems when we started. In the first instance, the use of the electron multiplier with the RGA was a source of trouble. We could not guarantee that the gain would remain the same during the course of an exposure, or from exposure to exposure. Therefore we reduced the pumping speed so we could run at higher pressure with the Faraday cup. Early on we had used an ion pump instead of the turbo; this proved to be very bad. The ion pump was the main source of background hydrogen in the system; furthermore, the amount of hydrogen coming from the pump was pressure-dependent. An ion gauge had been used to monitor total pressure, and this was finally removed also. Both of these devices produce low energy $x$ rays that in turn desorb hydrogen from the vacuum chamber walls.

This same setup was used to expose samples at temperatures near that of $\mathrm{LN}_{2}$. The 500-A feedthrough was made of a pure copper rod, roughly $1.5 \mathrm{~cm}$ in diameter. By putting the lower portion (the part exposed to air) in a bath of $\mathrm{LN}_{2}$, we were assured that the entire rod reached $\mathrm{LN}_{2}$ temperature. The sample, however, was in contact with the rod only by virtue of sitting upon it. The only way we had to measure the sample temperature was by removing the sample block from the vacuum chamber and measuring its temperature in air. This gave an upper limit to the temperature, which was found to be $120 \mathrm{~K}$. It is likely that the sample, in vacuum, was $100 \mathrm{~K}$ or below.

The procedures for measuring the electrodesorption from samples at or about liquid helium (LHe) temperatures were quite different. In this case the $e$-gun was put on the lower arm of the cross, firing upwards at the sample, which was attached to a stainless steel, cold finger filled with LHe. The moveable screen was brought in from the side. The samples were kept cold by using a high-conductivity grease to maintain good thermal conductivity between the sample and the copper bottom of the cold finger. Unlike the 
room-temperature case, the main pumping comes from the cold finger. When the cold exposures were being done, the turbo pump was valved off. In order to keep the electrodesorbed gas and the calibrated leak as symmetric as possible, the calibrated leak was moved to the place where the airlock had been. This did not provide perfect symmetry, but since the surface area of the cold finger was small compared to the area of the surrounding vacuum chamber, we assumed that the multiple bounces from room-temperature walls of the vacuum chamber would result in a substantially symmetric situation.

\subsection{MODEL FOR ELECTRODESORPTION}

The only information one gets from an electrodesorption exposure is the output of the RGA as a function of time. By calibrating the RGA response and keeping the beam size and current constant during the exposure, we find the amount of desorbed gas versus time. We were surprised to find that all of our sample exposures showed the same $1 / t$ response for sufficiently large $t$. It turns out that this behavior can be explained using a very simple model. If we assume that the surface in question has an amount $m$ of desorbable molecules per square centimeter, that there is a constant cross section $s$ for electrodesorption, and that there is a cylindrically symmetric beam with a Gaussian distribution of the form $j_{0} \exp -(r / R)^{2}$, where $j_{0}$ is the electron density in electrons $/ \mathrm{cm}^{2}$, then the number of desorbed molecules per second $M$ is given by the following expression:

$$
M=m \pi R^{2}\left(1-\exp \left[-\sigma j_{0} t\right]\right) / t
$$

When $t>1 / \sigma_{0}$ the above expression takes on the following form:

$$
M=m \pi R^{2} / t \text {. }
$$

Since we know the value of $R$ and $M(t)$, the long time behavior tells us directly the amount of desorbable gas $m$ on the surface. For short times with $t<<1 / \sigma_{0}$, we find $M(t)$ to be constant with a value

$$
M=m I \sigma \text {, }
$$

where $I$ is the total number of electrons/s hitting the sample. Since we measure $M$, and know $m$ from the long time behavior, this allows us to find a value for the cross section.

Support for the model was provided by several exposures of similar samples, but at different electron energies. The values of $m$ remained the same, although the cross section varied. These experiments were time-consuming to perform because the beam size would generally change at each voltage level and, therefore, it had to be remeasured for each sample. For this reason there were only a few measurements of $m$ versus electron energy. Another piece of support for the model was provided by the fact that the value of the cross section remained surprisingly constant when samples with very different values of $m$ were exposed.

Almost all of the exposures were done at an electron energy of $167 \mathrm{~V}$. Later, after many different samples had been tested, we measured the cross section versus voltage on an electroplated copper sample only.

\subsection{EXPERIMENTAL RESULTS}

The first goal of the program was to determine whether there were some copperplating methods that might have significantly less desorbable hydrogen than others. When the program started, there was considerable opinion among surface physicists and experts in related disciplines that the purity and size of grain boundaries, bulk hydrogen content, etc., would be the dominant factors in determining the amount of desorption. We learned several things quite early on. In the one instance, there was no relation between the amount of desorbable hydrogen and the amount of bulk hydrogen. There was also no relationship between the hydrogen outgassing rate and the desorbable hydrogen. 
In order to start with samples that would be as clean as possible, each sample was baked for $1 \mathrm{~h}$ in vacuum at $350 \mathrm{~K}$. Samples were then exposed to air, usually for no more than a few hours, before being inserted into the airlock. The results were remarkably uniform: the desorbable hydrogen varied from $4-5 \times 10^{15}$. The value for the cross section varied very little, with typical values of $(0.45 \pm 0.1) \times 10^{-17}$, and with the electron energy at $167 \mathrm{eV}$.

If the baked samples were left exposed to air for weeks or months, or if they were exposed to water, the amount of desorbable hydrogen increased to $15-50 \times 10^{15}$. The cross sections did not show a significant change from the values measured on the clean baked samples.

Since the samples contained considerable bulk hydrogen, it was thought that diffusion from the bulk, or possibly even surface diffusion, would cause a sample to become recontaminated. This was found not to be the case. A typical sample would be cleaned up during the course of a run, which might take 30 min or less. We would leave the sample in overnight and the next day expose it to the beam once more. The second exposure would give no increase in the amount of desorbed hydrogen above that of the dynamic background.

The dynamic background was assumed to result from secondary emissions caused by the sample striking the walls of the surrounding vacuum chamber. The troublesome part of this assumption is that it could not eliminate the possibility that some of the desorption was indeed from the sample, perhaps because of some mechanism such as stimulated diffusion. In order to investigate this problem, the electron beam was fired into a long, narrow tube, closed at the far end. Very little secondary emission was able to come back out of the entrance hole. Using this technique we were able to reduce the dynamic background to less than $1 \%$. This was essentially our noise level.

Since all the various copper samples did not satisfy the requirements for the SSC beam tube, we decided to look at various other coatings that might be applied. Because of the need to have very good surface conductivity, these coatings would have to be applied in very thin layers-i.e., less than a skin depth at $5 \mathrm{GHz}$, if their intrinsic conductivity weren't good enough. We didn't restrict ourselves to metals that may be easily plated on top of the copper. Instead we looked at a variety of metals that appeared to have very different properties to see if we could find anything that seemed to offer a substantial improvement. We tested gold, titanium, palladium, tin, platinum, and silver. There was not enough time to do these tests with a variety of surface treatments for each material; therefore, the values of $m$ should be regarded only as examples. It was surprising that the cross section for electrodesorption was, within experimental error, the same for all the different metals, namely $4-6 \times 10^{-17} \mathrm{~cm}^{2}$ for an electron beam energy of $167 \mathrm{eV}$. Typical values for $m$ were $16-20 \times 10^{15} \mathrm{~cm}^{-2}$. Silver had a high value of $80 \times 10^{15}$, and tin had a value of $8 \times 10^{15}$. Clean gold generally gave the best results, but when it was exposed to moisture, it would give values similar to the other metals. The ability of the metal surfaces to retain desorbable hydrogen depended in part on the metal's exposure to air. For instance, we tested pieces of OFHC copper as well as aluminum. The lowest values for $m$ were obtained when we would machine off a clean surface, and then within minutes insert the sample into the airlock. Values of $m$ as low as $3 \times 10^{15}$ were seen for both the copper and aluminum.

A series of measurements was made with the samples held at reduced temperatures. The first ones, as described in Section 3, were done at a temperature of 100-120 K. When the cold finger was cooled to $\mathrm{LN}_{2}$ temperatures, the water vapor component dropped by a factor of almost 100 . This was not unexpected. What was a surprise was the drop in the hydrogen partial pressure, which dropped by a factor of 2 or more over the course of $1 \mathrm{~h}$ or so. The source of this effect was unknown at the time, but could have been associated with a change in the RGA sensitivity. The experimental indications were that there was no more than a factor of 2 reduction in the desorption cross section. When we moved over to the cold finger, we had the option of filling it with either $\mathrm{LHe}_{\mathrm{H}} \mathrm{LN}_{2}$. We made measurements at both of these temperatures. We were unable to determine any reduction in the cold desorption cross sections. This was in agreement with earlier photodesorption experiments reported by Bintinger, Limon, and Rosenberg. 
We were also able to measure the cross section for electrodesorption of $\mathrm{H}_{2}$ that had condensed onto the face of the sample. The cross section was so large that we tended to saturate the RGA. We attempted to improve this situation by running at as low a current as possible. However, we couldn't make extreme changes without possibly changing other properties of the beam. We were able to put a lower limit on the cross section of about $33 \times 10^{-16} \mathrm{~cm}^{2}$ with an electron energy of $167 \mathrm{eV}$. We also obtained a value of $2 \times 10^{15} \mathrm{~cm}^{-2}$ for the surface coverage. We did not have time to get any data on the electrodesorption of the physisorbed hydrogen as a function of energy.

\subsection{CONCLUSIONS AND UNANSWERED QUESTIONS}

The principal conclusion of these studies is that there appears to be a layer of chemicals that forms on the surface of metals that is largely independent of what the metal is. The amounts of $\mathrm{CO}$ and $\mathrm{CO}_{2}$ relative to the amount of desorbed hydrogen are remarkably similar for all of the metals. In addition, it appears that the diffusion of hydrogen from the bulk, which may be a principal source of hydrogen outgassing, plays no role in determining the amount of electrodesorbed hydrogen.

All of the electrodesorption measurements were done at normal incidence. This assures that all of the surface is directly exposed to the electrons. If the electrons were incident at grazing incidence, then because of the surface roughness at the micron level we might expect large portions of the surface to be hidden from the beam. In a tube configuration it could be that the secondary emission that gave rise to our dynamic background would do an adequate job of exposing those parts of the pipe that would have been hidden from direct illumination. This would be an interesting area for future investigation.

At the time that the experimental program was shut down, we were investigating the reason for the fall of the hydrogen partial pressure when we cooled our cold finger to $\mathrm{LN}_{2}$ temperature. One of the tests we performed was to leak water vapor into the system for a few minutes. Then, when the leak was turned off, the water vapor would fairly rapidly return to its original pressure. The hydrogen would be found to be at an elevated level, which tended to be independent of how high the water pressure rose, and would remain at that level until the water vapor reached a certain level, at which point the hydrogen pressure would begin to drop, but at a much slower rate than the water vapor. The model we were developing to explain this behavior assumed that a certain fraction of the water molecules striking the surface would dissociate and form $\mathrm{H}^{+}$and $\mathrm{OH}^{-}$at the surface. The hydrogen would then diffuse into the bulk of the material, up to a certain critical concentration, and would find its way out as $\mathrm{H}_{2}$ through the same recombination mechanism as normal outgassing.

\subsection{ACKNOWLEDGMENTS}

This work could not have been accomplished without the continued advice and support of M. D. Pettigrew. He designed and procured most of the subsystems used for the experiments, and he operated and maintained them throughout the course of the work. I received much instruction and support, especially during the final months of the program, from Qing-Tang Jiang, a physicist who had previously been working with the UT/Arlington Surface Physics Group, but worked full-time at the SSC Laboratory after the funding ended at UT/A. 